\title{
Numerical Investigations of a New N-Body Simulation Method
}

\author{
E. Vilkoviskij \\ Fesenkov Astrophysical Institute, Observatory, Almaty, Kazakhstan \\ Email: vilk@aphi.kz
}

Received July 4, 2012; revised August 10, 2012; accepted August 17, 2012

\begin{abstract}
Numerical investigation of a new similarity method (the Aldar-Kose method) for N-body simulations is described. Using this method we have carried out numerical simulations for two tasks: 1) Calculation of the temporal behavior of different physical parameters of active galactic nuclei (AGN) containing a super massive black hole (SMBH), an accretion disk, and a compact stellar cluster; 2) Calculation of the stellar capture rate to the central SMBH without accretion disk. The calculations show good perspectives for applications of the similarity method to optimize the evolution model calculations of large stellar systems and of AGN.
\end{abstract}

Keywords: N-Body Simulations; Numerical Methods

\section{Introduction}

In the paper [1] a new similarity method (the Aldar-Kose method) for N-body simulations was proposed. Here we present numerical examples of the applications of this method to N-body simulations of AGN evolution, taking into account the star-disk dissipative interactions, and to simulations of the star capture rate to the central SMBH in AGN without accretion disk. The aim of the calculations is to determine the accuracy of the new method and its application limits.

Formally, the essence of the A-K method is simple and can be reduced to the following:

- A real stellar system (the "large" one with $N_{1}>10^{6}$ stars) is modeled as an ensemble of $m=N_{1} / N_{2}$ "small" systems, each containing $N_{2}=N_{1} / m$ gravitating particles. The initial conditions for the coordinates and velocities in the large and in each of the small systems are obtained from a random number procedure designed to imitate the initial quasi-equilibrium distribution functions of the stars-particles;

- The usual direct N-body simulations is provided for every member of the ensemble of $m=N_{1} / N_{2}$ small systems, and then (as is shown in [1]) the resulting physical characteristics of the large system can be obtained as those averaged over the ensemble of small systems;

- As shown in the paper [1], the resulting averaged values are equivalent to a single solution, obtained with the direct calculation for the large system, if all the solutions are taken at identical moments of the evolution time $T_{e v}$, equally defined in both the large and the small systems. This means that the physical characteristics at equal $T_{e v}$ are equal to the meansquare deviations, defined with the total number of stars in the large system.

It was shown also that the result is valid for dissipative systems as well, in particular, for AGN models, where the dissipative interactions of stars with the accretion disk are taken into account. In this case, the dissipative acceleration of a particle in the small systems has to be multiplied by a similarity scaling coefficient, $S C\left(N_{1}, N_{2}, T_{e v}\right)$ [1].

\section{Comparisons of the A-K Method with Direct N-Body Simulations of the AGN Dissipative Evolution}

With our computer we can perform direct N-body simulations for systems with maximum number of particles $N_{\max } \sim 32 \times 10^{3}=32 \mathrm{~K}$. But in real AGN, the typical total number of stars in the surrounding compact stellar clusters are about $N_{1} \sim 10^{6}-10^{9}$, which are $\sim(32-32 \mathrm{~K})$ times larger than our $N_{\max }$. So, to test the A-K method, we use the "ladder" approach to the comparison of model simulations with different $N_{2}<N_{1}$. The main idea of the approach is to substitute the comparison of the two systems with $N_{2} \ll N_{1}$ with the comparison of a sequence of systems with $N_{3}<N_{2} \cdots<N_{1}$. As deduced in [1], the inaccuracy of the calculations in the row is increased (from right to left!) non-linearly with $m_{i}=N_{i} / N_{i+1}$. So, if the accuracy of calculations of the $N_{2}$ system, using the 
A-K method with the ensemble of $m=N_{2} / N_{3}$ subsystems is acceptable, it (supposedly) will be acceptable for the representation of any larger systems with $N=m N_{2}=$ $m^{2} N_{3} \ldots$ particles and so on, up to $N_{1}$. This "Aldar-Kose axiom" needs to be established for any specific task to find the minimal number of particles $N_{2}(\mathrm{~min})$ in the systems which can safely (with an acceptable precision) be used in the ensemble of $m=N_{1} / N_{2}$, representing the larger $N_{1}$ system.

The main aims of our model simulations were as follows:

1) Comparison of the solutions obtained with "usual" direct N-body simulations for $N_{1}=32 \mathrm{~K}$ and $N_{1}=16 \mathrm{~K}$ to the solutions for the ensembles of $m$ systems with $N_{2}=$ $N_{1} / m$ (the A-K method);

2) Estimation of the dependences of the precision and time consumption of both methods from different model parameters.

We compared the following physical parameters of the models:

a) The growth rates of the central $\mathrm{BH}$ mass under different conditions;

b) The distribution functions of the stellar orbits' inclination angles $(\cos (i))$ in two regions, one inside the size of the accretion disk and the other in the whole stellar system;

c) The distribution functions of the particle orbits' eccentricities $e=\left[1+E^{2} L^{2} /\left(m+M_{b h}\right)^{2}\right]$ of the stars (particles) in the same regions $\left(E=v^{2} / 2-\left(m+M_{b h}\right) / r\right.$ is the energy and $L$ is the angular momentum of a star in the gravitational field of the $\mathrm{BH}$ ).

The comparisons were performed for the models with two different rates of growth of the central SMBH (pure stellar accretion and "stellar plus gas" accretion rates), to compare the evolution of the system in the cases. (Both cases are presented with some "toy" models, acceptable for the formal testing of two different accretions rate cases only.)

The following simulations were fulfilled and compared to each other:

A. The direct N-body simulations for the particle numbers $N_{1}=32 \mathrm{~K}$ and $16 \mathrm{~K}$ (in the figures below the cases are labeled as $1 \times 32 \mathrm{~K}$ and $1 \times 16 \mathrm{~K})$.

B. The model simulations using the $\mathbf{A}-\mathbf{K}$ method for the same systems as the result of averaging over $m=N_{1} / N_{2}$ "small" systems with different $m$ values (labeled in the figures as $m \times N_{2}$ ).

The results of the calculations are presented in Figures 1-8. As it was shown in [1], one has to compare parameters of the systems in the equivalent moments of evolution, the "evolution times" $T_{e v}(t)>t / T_{r x}(t=0)$ due to variable (diminishing due to accretion and evaporation) particle numbers. The larger is the evolution time, the bigger the accuracy gain with this correction.

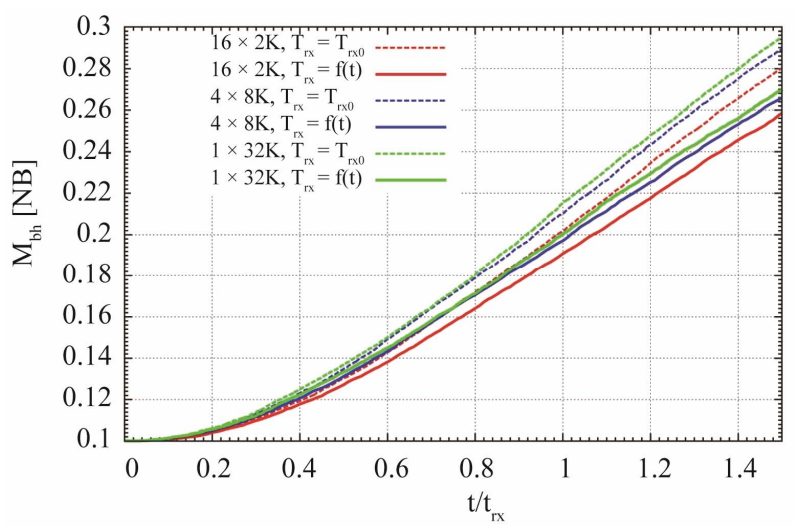

Figure 1. Evolution of the black hole mass due to the accretion of stars. The dashed (upper) lines represent the case $T_{r x}=$ const, and solid lines represent the case with the timedependent $T_{r x}(t)$ (i.e., the BH mass at the $T_{e v}(t)$ moments). The green lines are for direct calculation $(m=1)$. Results of the A-K method are shown with blue lines for $m=4$, and with red lines for $m=16$ representing systems.

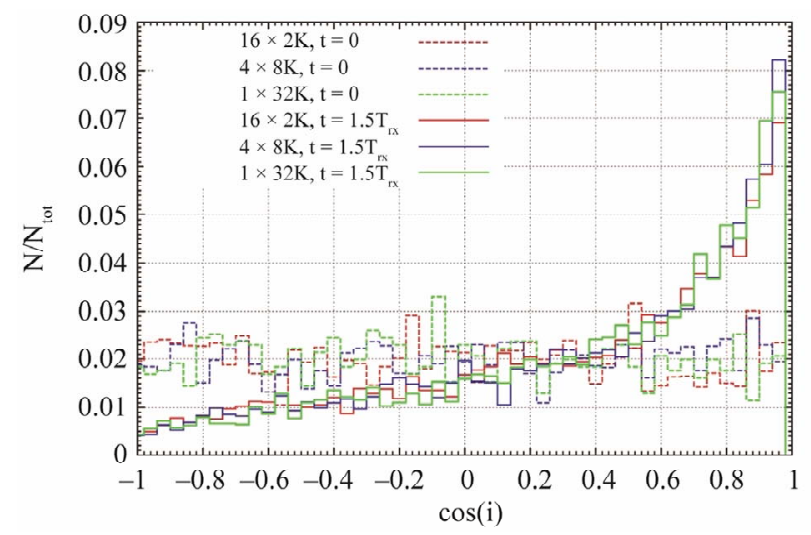

Figure 2. Distribution of inclination cosines for inner stars' orbits in 3 simulations at the moments $t=0$ (dashed lines) and $t=1.5 T_{r x}(t)$ (solid lines). Green lines are for direct $32 \mathrm{~K}$ $\mathrm{N}$-body simulation, and blue and red ones are for the A-K method with $m=4$ and $m=16$.

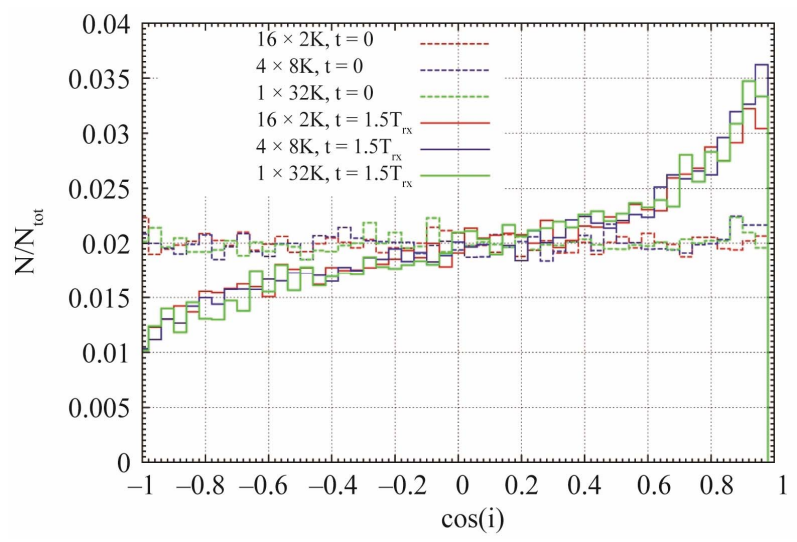

Figure 3. Distribution of inclination cosines for all stars in three simulations with different numbers $m$ of systems in A-K ensemble (notations are as in Figure 2). 


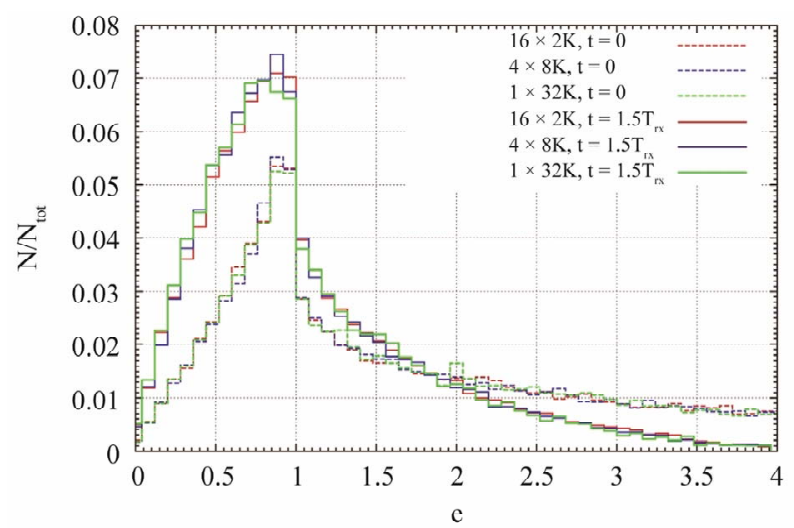

Figure 4. Distribution of eccentricities for all stars (notations are as in Figure 2).

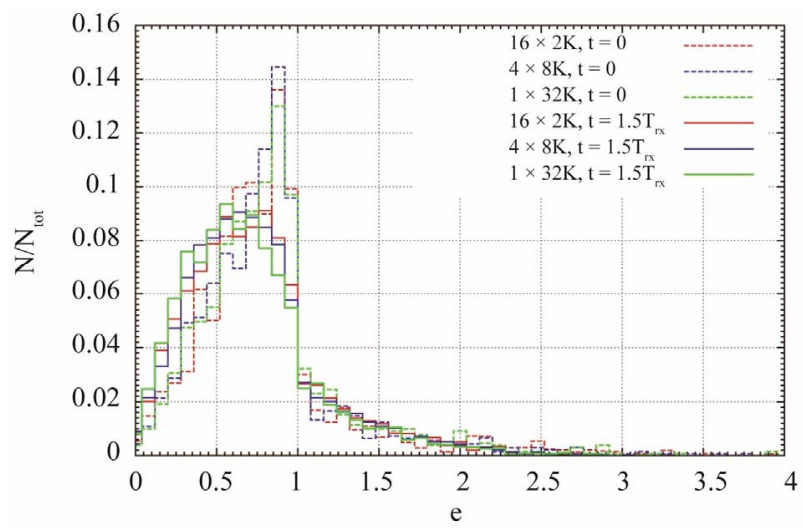

Figure 5. Distribution of eccentricities for inner stars (notations are as in Figure 2).

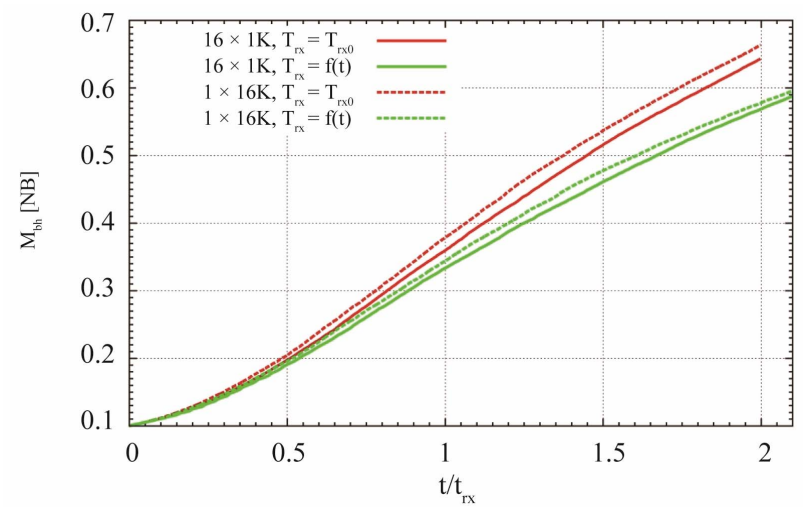

Figure 6. Growth of the SMBH mass due to both stellar and gas accretion, obtained with direct simulations (dashed lines) and A-K method (smooth lines). Green lines present more exact dependences on $T_{e v}$.

In Figures 1-5 the evolution of physical parameters is presented, supposing that the SMBH mass is growing as the mass of the stars, crossing some "accretion radius" $R_{a c}=$ 0.01. The foundation of the supposition is the inflow of stars to the center of the AD due to star-disk interactions [2]. Though the stellar capture rate to the central $\mathrm{BH}$ can

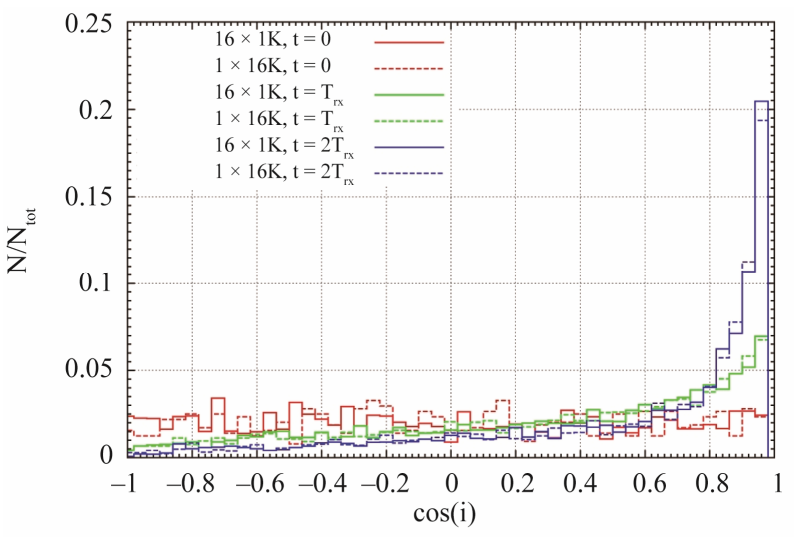

Figure 7. Distribution of inclination cosines for inner stars at three moments of evolution, $T_{e v}=0$ (red), $T_{e v}=1$ (green) and $T_{e v}=2$ (blue), calculated with the direct (broken lines) and the A-K (solid lines) methods.

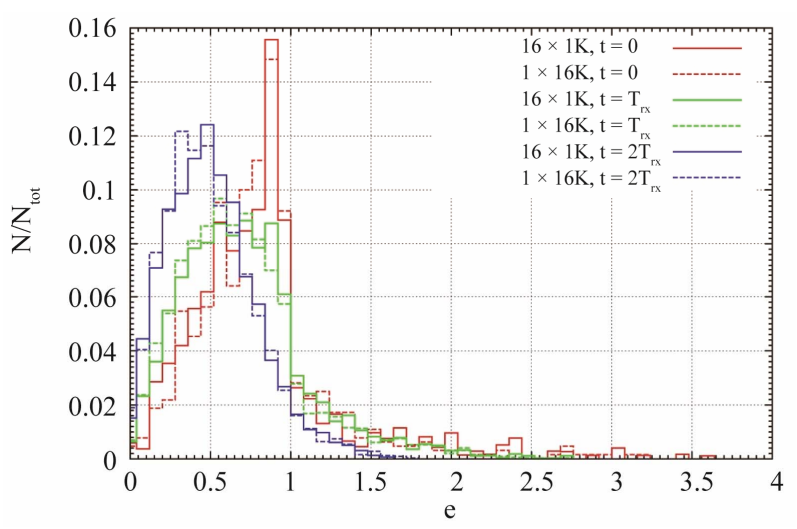

Figure 8. Distribution of eccentricities for inner stars with the gas accretion switched on. Notations are as in Figure 7.

be diminished with the process of "melting" of the stars close to the $\mathrm{BH}$ due to the star-disk interactions [2], in the present paper we permit the arbitrary suppositions about the stellar and gas accretion rates just for the sake of a formal testing of the new N-body solution methods with different growth rates of the central BH. (The more realistic calculations of the $M_{b h}(t)$ behavior would demand much more detailed model calculations of the star-disk interactions, which will be done in following works.)

In Figure 1 the difference of the dashed and solid green lines is "artificial" (due to different scales of the $t / t_{r x}$ axes, the dashed line for $t_{r x}=$ const, and the solid one for $t_{r x}=$ $f(t))$. But the differences of the lines with different colors are real, depending on the A-K approximation defined with $m$ representing "small" systems. One can see that the deviations of the blue and red lines (the A-K calculations with different $m$ ) from the green lines (the direct calculations) are slightly smaller for solid lines (the time-dependent relaxation time units). In this case deviations are the real characteristics of the A-K precision. One can see that the deviations of the A-K method from the direct 
simulation $(1 \times 32 \mathrm{~K}$, the green lines $)$ are in the both cases smaller for $m=4\left(N_{2}=8 \mathrm{~K}\right.$, blue lines) and larger for $m=$ $16\left(N_{2}=2 \mathrm{~K}\right.$, red lines), where $m$ is the number of the representing systems in the A-K ensemble. So, $N_{2}=8 \mathrm{~K}$ can be taken as the minimal number of particles in the small systems to represent the $N_{1}=32 \mathrm{~K}$ large system, and (admittedly) to represent any $N>N_{1}$ system with $m=N / N_{2}$ subsystems having each $N_{2}$ particles. We also find out that both the inaccuracy and the duration of the A-K simulations are strongly increased with $N<N_{\min }=2$ $\mathrm{K}$ (that is $m>16$ ).

The calculated distributions of cosines of the orbital inclination angle's to the accretion disc plane, and the distributions of particle's eccentricities both for the inner orbits (in the region of size equal to the accretion disk size) and for the whole system are shown in Figures 2-5.

To check the A-K method in more details, we varied the mass accretion rate. For the case presented in Figures 1-5, we supposed that all growth of the SMBH mass is due to stellar captures only (the absence of the gas inflow from the gas disc to the black hole is an artificial admission, acceptable for the formal testing of our tasks only). Below we show results of investigations of another case: The SMBH mass is increased with both the accreted gas and captured stars (which supposedly are the stars crossing the sphere with radius $R=0.01$ ). In that case the gas supply during one relaxation time was supposed to gain mass $\Delta M=$ $M_{b h 0}$, where $M_{b h 0}$ is the initial mass of the black hole $\left(M_{b h 0}=\right.$ $0.1 \mathrm{NBU}$ in our model). The results of the calculations are shown in Figures 6-8.

One can see from Figures $\mathbf{7}$ and $\mathbf{8}$ that in this case (increased mass inflow to the SMBH) the stellar orbits tend to be more circular in the inner region of the system, and eccentricities are diminished due to the increased $\mathrm{BH}$ mass. The A-K results are presented with solid lines, and direct calculations with dashed lines; both go close to each other.

\section{Application of the A-K Method to N-Body Simulations of AGN without Accretion Disk}

Another interesting task is calculations of the star capture rates to the central SMBH without accretion disk in the stellar systems with zero rotational moment. This drastically diminishes the accretion rate of the stars. The reason is that the disruption radii (DR) of stars by the central SMBH are small and the "loss cone" (the region of the orbits inside the DR) has to be filled by the stars' moment diffusion [3]. We adopt DR $=8.0 \mathrm{e}-07$ from [4] to calculate the stellar capture rate using the A-K method. The total number of stars in the system was taken $N_{1}=10^{5}$ and the central $B H$ mass $M_{B H}=0.01$ (as in the [4]), but in our case the initial structure of the stellar system is defined with the Plummer distribution.
As the direct N-body simulation of $N=10^{5}$ particles would take too much time with our computer, we performed the simulations using the A-K method only, that is the simulations of evolution of the ensemble of $m=50$ "small" stellar systems with the central BH (each containing $N_{2}=N_{1} / m=2 \mathrm{~K}$ stars-particles), representing the evolution of the "large" systems with $N_{1}=100 \mathrm{~K}$ equalmass stars, surrounding the SMBH with $M_{B H}=0.01$ (one percent of the stellar system's mass in NBU, as was supposed in [4]). We choose the "capture radius" of stars to the central $B H r_{c a p}=\mathrm{DR}=8 \mathrm{e}-7$ to compare our result to that of [4]. The results of calculations are shown in Figures 9 and 10.

As seen from Figure 9, each individual run with $N=2$ $\mathrm{K}$ particles yields a few accretion events, presented with vertical "jumps" at the ladder-like thin lines. In every capture event, the BH mass increases by $m=N_{1} / N_{2}=50$ stars, equal to $5 \times 10^{-2}$ of the initial $\mathrm{BH}$ mass, which gives the jumps. We had 15 colors only to draw the 50 "laddertracks", so the resulting picture is a very tangled one. But averaging over 50 such runs (the A-K method) produces a rather smooth curve (shown with the red thick line) with small enough fluctuations, since it is equal to one run with $100 \mathrm{~K}$ particles, in accordance with the A-K paradigm.

In the Figure 9, each ladder-like track represents an evolution of the BH mass in each of the "small" subsystem of the ensemble. The deviations from the average thick red line characterize the mean-square deviations of the individual evolution tracks of the small systems. One can see that in spite of the large deviations in the behavior of the "small" subsystems of the A-K ensemble, the averaged curve remains smooth enough.

We remind that in the Figures $\mathbf{9}$ and $\mathbf{1 0}$ the time scale is presented in units of the "evolution time", $T_{e v}=t / t_{r x}$, equally defined for the systems with any different numbers of particles (see [1]), which permits to plot and compare them at the same pictures, where the essence of A-K approach is quite visible.

Figure 10 shows how the fluctuations vary with different numbers of runs $m$, taken for averaging, which permits comparison of the "full" (complete) A-K simulation (red line) with the "truncated" A-K simulations, averaged correspondently over 30 (green line), 10 (blue) and 5 (agenda) representing subsystems. The average slope of the lines (well visible in the red and green lines) looks evidently increased at the moment of evolution time close to one relaxation time, possibly as a result of a cusp formation in the stellar system. Note, that all the "evolution tracks" are close enough at the moments of the evolution time $T_{e v}=t / t_{r x} \sim 1.5-2$, which points to possible application of the "truncated" A-K method for the snap-shot estimations of the large stellar system evolution rate.

From this result, we have calculated the star capture rates $\mathrm{CR}$ per $\mathrm{N}$-body time unit (one crossing time, as was 


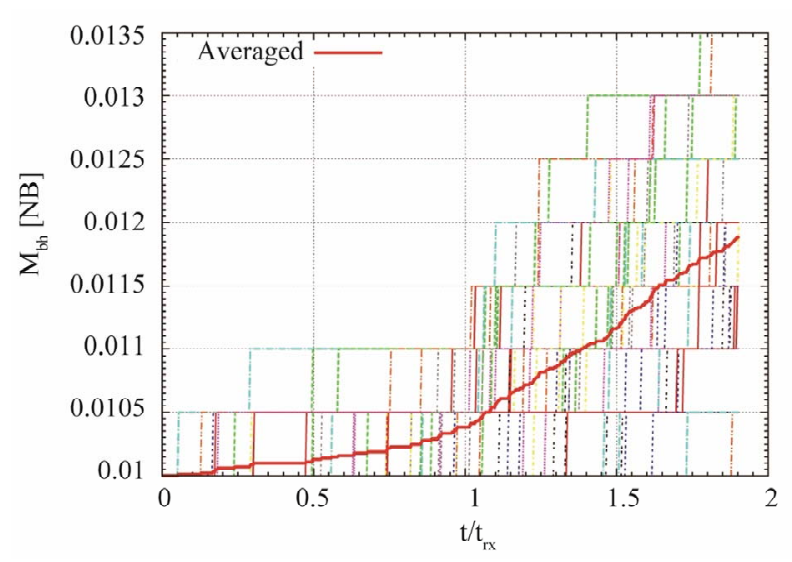

Figure 9. The evolution track of the black hole mass (red thick line) averaged over 50 runs (which is equal to the result of $N_{1}=100 \mathrm{~K}$ particles evolution), as compared to the evolution tracks of each of the small systems of the ensemble with $N_{2}=2 \mathrm{~K}$ particles in each (the thin ladder-like lines of different colors).

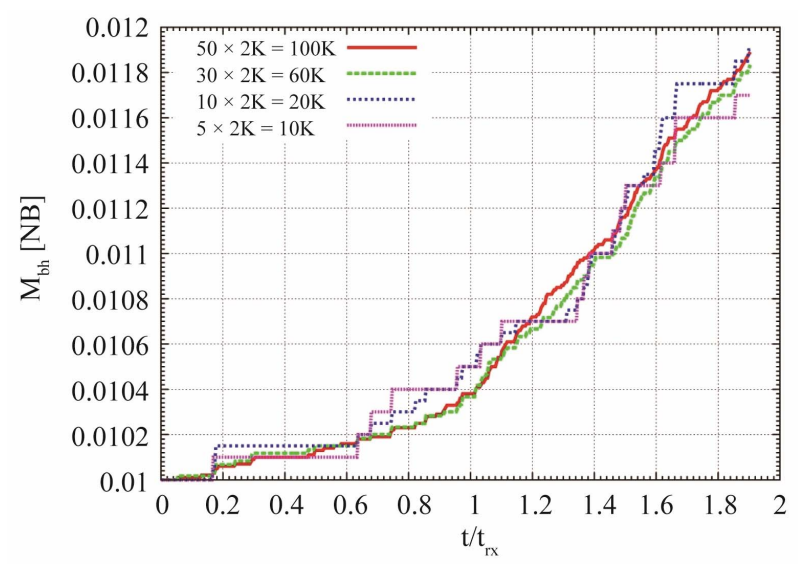

Figure 10. The evolution of the black hole mass, averaged over different numbers of runs $m$ (each run with $N_{2}=2 \mathrm{~K}$ ).

done in [4]). We obtain CR 1.5 stars $/ t_{c r}$ in the time interval $T<1 T_{e v}$ and CR $\sim 6$ stars $/ t_{c r}$ at $T>1 T_{e v}$. The first result is close enough to the result obtained in [4] with the direct N-body simulations for the same particle number $(N=$ $10^{5}$ ) and time interval smaller than $T<T_{e v}$.

For our full A-K variant $(m=50)$ we spent $t \sim 83$ hours with the GPU computer (using 2 Tesla cards and CPU 2.5 $\mathrm{GHz})$, and for the "truncated" $(m=5)$ case we spent $t \sim 8.3$ hours only. The direct N-body simulations of $N=10^{5}$ particles with our computer would demand calculation time $t>1$ year.

\section{Conclusions}

To estimate possible applications of the new similarity method (the A-K method), suggested in [1], we have performed simulations of two physically different model tasks:
1) We use the A-K method to compare the direct simulations of the $N_{1}=32 \mathrm{~K}$ and $N_{1}=16 \mathrm{~K}$ (labeled in the Figures 1-8 as $1 \times 32$ and $1 \times 16$ ) to the calculations with the A-K method, using different numbers $m$ of small systems in the ensembles $(m=2,4)$ (labeled in Figures as $2 \times 16,4 \times 8$ and $2 \times 8,4 \times 4)$. We show that the calculation error increases with increasing $m$, and conclude that particle numbers around $N_{2} \geq 4 \mathrm{~K}$ in the A-K modeled "small" systems (representing any larger $N_{1}$ systems) are enough to calculate the real evolution of AGN containing the compact stellar clusters with $N_{1} \geq 30 \mathrm{~K}$ stars and with the masses of the accretion disks $M_{d} \leq 0.01 M_{1}$. To obtain more realistic lower limit of $N_{2}$ and upper limits of $N_{1}$, the direct calculations of the models with larger $N_{1}$ have to be fulfilled;

2) We use the A-K method to calculate the star capture rates by the central SMBH for the case where there is no accretion disk in the stellar system with zero rotational moment. The A-K method in this case seems to give results close to those obtained with the direct simulations [4]. The scaling problem of the task (see [5]) needs addition investigations.

All simulations were performed with the phiGRAPE + GPU code [6].

Our main preliminary conclusions about possibilities of the A-K method are quite optimistic, though the method certainly needs further investigations and testing with more advanced computers. The preliminary results of our investigation promise good prospects of the A-K method applications to the calculation of various evolution scenarios of AGNs with compact stellar clusters and to other evolution models of large stellar systems.

We hope that the A-K method will open new ways to more detailed physical models of stellar systems and AGN evolution due to economy of time for the pure stellar-dynamical calculation.

\section{REFERENCES}

[1] E. Vilkoviskij, "On the New Method of N-Body Simulations," IJAA, 2012, in Press.

[2] E. Vilkoviskij and B. Czerny, "The Role of the Central Stellar Cluster in AGN," Astronomy and Astrophysics, Vol. 387, No. 3, 2002, pp. 804-819. doi:10.1051/0004-6361:20020255

[3] J. Frank and M. Rees, "Effects of Massive Central Black Holes on Dense Stellar Systems," Monthly Notices of the Royal Astronomical Society, Vol. 176, 1976, pp. 633-647.

[4] M. Brockamp, H. Baumgardt and P. Kroupa, “Tidal Disruption Rate of Stars by Supermassive Black Holes Obtained by Direct N-Body Simulations," arXiv: 1108.2270v1, 2011.

[5] S. Aarseth and D. Heggie, "Basic N-Body Modelling of the Evolution of Globular Clusters. I. Time Scaling," arXiv: astro-ph /9805344V1, 1998. 
[6] S. Harfst, A. Gualandris, D. Merritt, R. Spurzem, S. P. Zwart and P. Berczik, "Performance Analysis of Direct NBody Algorithms on Special-Purpose Supercomputers,"
New Astronomy, Vol. 12, No. 5, 2007, pp. 357-377. doi:10.1016/j.newast.2006.11.003 